\title{
Performance ANAlysis of Upsample Filter FOR SAMPLE RATE CONVERTER
}

\author{
Manish Sabraj ${ }^{1}$ and Vipan Kakkar $^{2}$ \\ ${ }^{1,2}$ School of Electronics \& Communication Engg. \\ Shri Mata Vaishno Devi University, Jammu and Kashmir (India) \\ manishsabrajeyahoo.co.in \\ vipan.kakaresmvdu.ac.in
}

\begin{abstract}
Now a days many signal processing tasks are performed in the digital domain. Various sample rates are used on the basis of required signal quality and the available bandwidth. Sample-rate conversion is therefore inevitable to interface systems with different sample rates. The aim of digital sample rate conversion is to bring a digital audio signal from one sample frequency to another. Some information is lost while sampling.The distortion of the audio signal introduced by the sample rate converter should be as low as possible. The generation of the output samples from the input samples may be performed by the application of various techniques, In this paper, a new technique of digital sample-rate converter is proposed. We discuss performance analysis of upsample filter in proposed digital sample rate converter.
\end{abstract}

\section{KEYWORDS}

Sample Rate Converter, Conversion Factor, Upsample-Downsample Filter, Sigma-Delta Modulator, Frequency Detector.

\section{INTRODUCTION}

The need for inexpensive,reduced implementation complexity, high resolution analog-to-digital converters (ADCs) in digital audio has led to the use of oversampling techniques in the design of such conveters rather than the traditional successive-approximation technique [1-3]. For example, by oversampling the quantization noise inherent in ADCs is spread over a wider frequency range, so that the in-band noise is made small, effectively increasing the number of ADC bits. Further, these new ranges of high performance ADCs employ delta sigma modulation because of its simplicity. Most, if not all, the inexpensive, high resolution $\operatorname{ADCs}(18,20,24$ bits) in use today employ multirate processing.

In this paper, analysis results are presented for a new method of digital sample rate converter [4]. The operation principle of the new technique of sample rate conversion is very simple. An input sample is directly transferred to the output, while per unit of time, a certain amount of these samples is mislaid or repeated, depending on the difference in input and output sample frequencies. The omission, acceptance or repetition of a sample is called 'validation'. In order to get the simplest hardware implementation, the choice has been made to use only the take-over operation and the repetition process in the current system solution. This means that the output sampling frequency of the sample rate converter is always larger than the input sample frequency. The effectiveness of the proposed procedure in terms of memory and MIPS usage for SRC with

DOI : 10.5121/sipij.2012.3105 
other existing methods has been described. This method can also be extended for sampling rate conversion from DAT to CD. The proposed procedure can be easily implemented in a DSP for a real-time sampling rate conversion

The process of repeating samples unsurprisingly introduces errors. The resulting output samples will have correct values, but as a result of the validation process, they are placed on the output time grid with a variable time delay with respect to the input time grid. As a result, the output sequence should be observed as the input sequence, having the correct signal amplitude, which is sampled at erroneous time moments. The effect is the same as sampling the input signal by a jittered clock [5]. As a result, it can be stated that the time error mechanism introduced by the validation method is time jitter.

If all input samples would be transferred to the output grid without the repetition or omission of a certain amount of them, then the output signal would be just a delayed version of the input signal, exhibiting the same shape. It is the repetition and omission (in the current system setup nly the repetition ) of input samples that give rise to a variation in time delay for each individual output sample. This variation in individual time delays introduces phase errors. As a result of this, the shape of the output signal will be distorted [6].

The time errors introduced by the conversion process can be reduced considerably by applying upsampling and downsampling techniques. The input sample rate of the converter will be higher so that the conversion errors are smaller, resulting in smaller time jitter. These techniques do not serve when we want to achieve the very high analog audio performance required for specialized applications [7]. By using a sigma-delta modulator (noise shaper) as control source for the conversion process, the time errors will be shaped to the higher frequency region. As a result, the audio quality ( in the baseband) of the signal will be preserved, provided that sufficient bandwidth is created by upsampling of the input signal. The high frequency (out of base band) phase modulation terms can be filtered by a decimation filter or an analog low-pass filter which is directly placed after the sample-rate converter [8]. Figure 1 shows the block diagram of the complete sample-rate converter.

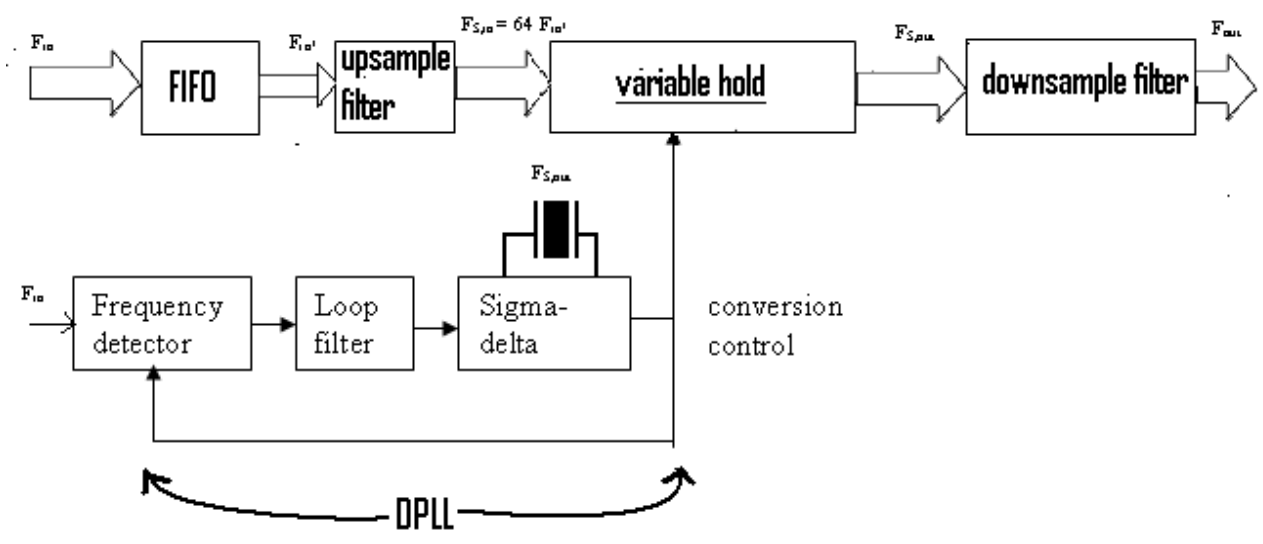

Fig. 1. Block diagram of the sample-rate converter.

As has already been mentioned, only the input sample take over operation will be employed here in order to get the simplest hardware. This means that the input sample frequency of the converter must be always be smaller than the output sample frequency. With this constraint imposed, it is assured that all input samples are used in the output sequence, none of them being omitted. The 
extra output samples per unit of time are introduced in the output sequence by repetition of their prior output samples.

\section{GUIDELINES For UPSAMPLE FILTER}

The upsample filter shown in figure 1 generates the signal for variable hold function. The upsample filter consists of an upsampler and a low pass filter. The upsampler inserts N-1 samples with zero amplitude in the input sequence (sampling frequency $\mathrm{F}_{\text {in }}{ }^{*}$ ), causing an increase of the fundamental frequency interval of the input sequence from $\mathrm{F}_{\mathrm{in}}{ }^{*}$ to $\mathrm{NF}_{\mathrm{in}}{ }^{*}$. Because sampling and upsampling add spectral images around the sampling frequency and multiples of it, these high frequency spectral images must be suppressed by a low-pass filter.

This low-pass filter, which operates on the new sampling frequency, has a limited stopband attenuation which means that there are residual spectral images (having a small amplitude) in the high-frequency band. This implies that for the spectral representation of the input sinewave, the high-frequency low-amplitude mirror spectra (also to be modeled by delta functions) must be added to the delta functions for sinewave. The high frequency mirror spectra cause the bandwidth of the input signal to increase.

The spectral representation of the sample-rate conversion process given by following equation can be used to establish the required stopband attenuation of the upsample filter.

$S_{y y}(f)=S_{x x}(f)+\frac{A^{2} f_{\text {in }}^{2}}{F_{S, \text { out }}^{2}} \cdot\left(1-\frac{F_{S, \text { in }}}{F_{S, \text { out }}}\right)^{2} \cdot\left[\begin{array}{l}\frac{1}{\left(f-f_{\text {in }}\right)^{2}} \cdot S_{S D S D}\left(f-f_{\text {in }}\right)+ \\ \frac{1}{\left(f+f_{\text {in })}\right)^{2}} \cdot S_{S D S D}\left(f+f_{\text {in }}\right)\end{array}\right]$

When the dependency on the time error spectrum is considered instead of the sigma-delta spectrum, the following equation is found:

$S_{y y}(f)=S_{x x}(f)+2 \pi^{2} A^{2} f_{i n}^{2} \cdot\left[S_{\Delta t \Delta t}\left(f-f_{\text {in }}\right)+S_{\Delta t \Delta t}\left(f+f_{\text {in }}\right)\right]$

The frequency $f_{\text {in }}$ in (2) denotes the input frequency of the sinewave in the audio band (i.e. not the sampling frequency).

With the upsample filter taken into account, the input signal consists of this sinewave together with the additional high-frequency mirror components. When the time error spectrum contains a discrete spectral peak at a frequency within $20 \mathrm{kHz}$ near such a mirror component, then the phase modulation term at the difference frequency falls into the audio baseband. The amplitude of such a modulation term is, according to (2), proportional to the frequency of the mirror component, the amplitude of this mirror component and the amplitude of the time error component.

In order to establish the required stopband attenuation of the low-pass filter, the largest modulation terms that can fall into the audio base band, have to be found. This is done by examining the time error spectrum for discrete peaks. This time error spectrum has a discrete has a discrete peak for the input sampling frequency (largest amplitude), and multiples of it. The fundamental frequency interval of this time error spectrum is equal to the output signal and amounts $\mathrm{F}_{\mathrm{S} \text {,out }} / 2$. The fundamental frequency interval of the input signal is $\mathrm{F}_{\mathrm{S}, \mathrm{in}} / 2$, which is smaller.

For digital audio the mostly used sample frequencies fall in the range from $32 \mathrm{kHz}-5 \%$ to 48 $\mathrm{kHz}+5 \%$. This means that the input sampling frequency of the total sample-rate converter ranges 
from $30.4 \mathrm{kHz}$ to $50.4 \mathrm{kHz}$. The input sampling frequency $F_{\mathrm{S} \text {,in }}$ of the actual sample-rate converter ranges from $1.9456 \mathrm{MHz}$ to $3.2256 \mathrm{MHz}$ (64 times upsampled). The output sampling frequency is fixed at $128 \mathrm{~F}_{\mathrm{s}}=5.6448 \mathrm{MHz}$.

For an input frequency of $30.4 \mathrm{kHz}$, the fundamental interval of the input signal amounts 0.9728 $\mathrm{MHz}$ while for an input sampling frequency of $50.4 \mathrm{kHz}$ this value is $1.6128 \mathrm{MHz}$. This means that the highest frequency component of the input spectrum that can generate modulation terms in the audio baseband is for a $30.4 \mathrm{kHz}$ input sampling frequency equal to $972.8 \mathrm{kHz}+20$ $\mathrm{kHz}=992.8 \mathrm{kHz}$. For a $50.4 \mathrm{kHz}$ input sampling frequency this highest frequency amounts 1612.8 $\mathrm{kHz}+20 \mathrm{kHz}=1632.8 \mathrm{kHz}$. The first spectral peak at the input sampling frequency $\mathrm{F}_{\mathrm{S}, \mathrm{in}}$ in the time error spectrum, which has the largest amplitude, cannot generate modulation terms in the audio baseband. Of the second and higher-order spectral peaks that fold back to frequencies below the highest frequency the peaks with the largest modulation product have to be found. The largest modulation product will be reached for the spectral peaks with the maximum frequency and the maximum amplitude.

Table 1 lists the largest intermodulation terms for the considered range of input sample frequencies, using a third order sample-rate converter. The values in the last column are obtained using the difference frequency term in (2). The frequency $f_{\text {in }}$ in (2) denotes the frequency of the spectral image in the high-frequency band. It appears from table 1 that for an input sampling frequency of $50.4 \mathrm{kHz}$, a $0 \mathrm{~dB}$ mirror component (amplitude $\mathrm{A}=1$ [Volt]) would give rise to a baseband modulation term with an amplitude of $-22.8 \mathrm{~dB}$, which is worst case in the considered frequency range. The required stopband attenuation of the upsample filter is found by considering the performance degradation of the sample-rate converter. An input sampling frequency of 50.4 $\mathrm{kHz}$ (internal input sampling frequency of $3.2 \mathrm{MHz}$ ) corresponds to a conversion factor of 1.75 .

Table 1 Largest modulation terms falling into the baseband for a $0 \mathrm{~dB}$ spectral image

\begin{tabular}{|c|c|c|c|c|c|c|}
\hline $\begin{array}{l}\text { Input } \\
\text { sampling } \\
\text { frequency } \\
\text { of the } \\
\text { total } \\
\text { converter } \\
{[\mathrm{kHz}]}\end{array}$ & $\begin{array}{l}\text { Internal } \\
\text { input } \\
\text { sampling } \\
\text { frequency } \\
\mathrm{F}_{\mathrm{s}, \mathrm{in}}\left[\mathrm{xF}_{\mathrm{s}}\right]\end{array}$ & $\begin{array}{l}\text { Fundamental } \\
\text { frequency } \\
\text { interval of } \\
\text { the } \\
\text { upsampled } \\
\text { input signal } \\
\text { [kHz] }\end{array}$ & $\begin{array}{l}\text { Frequency } \\
\text { spectral } \\
\text { peak in } \\
\text { time error } \\
\text { spectrum } \\
{[\mathrm{kHz}]}\end{array}$ & $\begin{array}{l}\text { Amplitude } \\
\text { of spectral } \\
\text { peak }[\mathrm{dB}]\end{array}$ & $\begin{array}{l}\text { Amplitude } \\
\text { of spectral } \\
\text { peak } \\
{\left[\text { Volt }^{2}\right]}\end{array}$ & $\begin{array}{l}\text { Amplitude } \\
\text { of } \\
\text { baseband } \\
\text { modulation } \\
\text { term for a } \\
\text { OdB } \\
\text { mirror } \\
\text { component } \\
\text { [dB] }\end{array}$ \\
\hline 30.401 & 44.12 & 972.832 & 736.5 & -163.5 & $4.47 \times 10^{-17}$ & -33.2 \\
\hline 32.001 & 46.44 & 1024.032 & 499.4 & -161.5 & $7.08 \times 10^{-17}$ & -34.6 \\
\hline 40.401 & 58.63 & 1292.832 & 473.5 & -152.1 & $6.17 \times 10^{-16}$ & -25.6 \\
\hline 48.001 & 69.66 & 1536.032 & 499.3 & -153.7 & $4.27 \times 10^{-16}$ & -26.8 \\
\hline 50.401 & 73.14 & 1612.832 & 806.5 & -153.9 & $4.07 \times 10^{-16}$ & -22.8 \\
\hline
\end{tabular}

It is observed that for a conversion factor of 1.75 the worst case SNR of the sample-rate converter - without upsample filter - is about $98 \mathrm{~dB}$. The required stopband attenuation must be $98 \mathrm{~dB}-$ $22.8 \mathrm{~dB}=75.2 \mathrm{~dB}$ so that the same performance loss is obtained as in the situation without the upsample filter. 


\section{Simulation and Performance Analysis}

The total sample-rate converter including an upsample filter has been simulated. The upsample filter, which has a stopband suppression of $75 \mathrm{~dB}$, has been designed by means of the program, which is a part of simulation. The worst case situation is considered for a third-order sample-rate converter. The input sampling frequency amounts $50.4 \mathrm{kHz}$ which corresponds to $48 \mathrm{kHz}+5 \%$ while the frequency of input sinewave is $20 \mathrm{kHz}$. The input signal is 64 times upsampled by the upsample filter which consists of a cascade of six sections. Each of these sections upsamples the input signal with a factor two.The first filter section has to generate a fairly steep transition from the passband to the stopband which implies a large number of filter taps ( coefficients). The following filter sections have consequently a much more relaxed transistion band which implies that the number of filter taps can be much smaller for these sections. The upsample filter has been designed this way in order to minimize the total number of calculations during the simulation.

The filter transfer function is dependent on the input sampling frequency. When the filter transfer function is optimized for an input sampling frequency of $44.1 \mathrm{kHz}$, then the transition band ranges from $20 \mathrm{kHz}$ to $22.05 \mathrm{kHz}$. When the same filter coefficients are used for an input sampling frequency of $32 \mathrm{kHz}$, the frequencies marking the transition band are linearly scaled and become $14.51 \mathrm{kHz}$ and $16 \mathrm{kHz}$ respectively. This means that the bandwidth of the output audio signal is limited to $14.5 \mathrm{kHz}$ and consequently it will not be suited for high-end applications. This makes clear that one should be careful with the choice of the upsample filter.

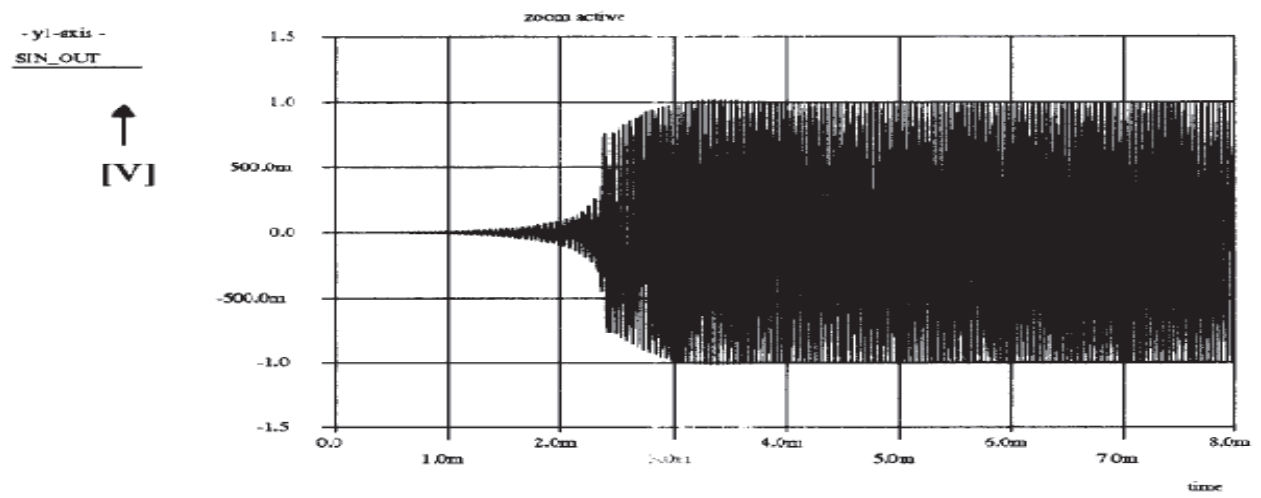

Fig. 2 Transient response of the total sample-rate converter to an input sinewave with a frequency of $20 \mathrm{kHz} ; \mathrm{F}_{\mathrm{in}}=50.4 \mathrm{kHz}, \mathrm{F}_{\mathrm{S}, \text { out }}=5.6448 \mathrm{MHz}$.

Figure 2 shows the transient response of the sample-rate converter to an input sinewave with a frequency of $20 \mathrm{kHz}$. It can be seen that, due to the delay in the filter sections, it takes a few milliseconds before the amplitude of the output signal has settled.

In figure 3 the frequency domain presentations of the output signal of a third-order sample-rate converter without upsample filter (above) and with upsample filter (below) are compared. For both results the number of simulation points is 65536 . The FFT is taken of the transient response for the time interval between $3 \mathrm{msec}$ and $11.61 \mathrm{msec}$, so that the amplitude of the output signal has settled (figure 2).

The two frequency plots look quite similar. It appears that the average noise level in the audio baseband is with use of the upsample filter about $-120 \mathrm{~dB}$, which is about the same as in the situation without upsample filter. The signal-to-noise ratio for a frequency range from $100 \mathrm{~Hz}$ to $20 \mathrm{kHz}$ is for the situation without upsample filter $98.79 \mathrm{~dB}$ where a SNR of $98.69 \mathrm{~dB}$ is 
measured for the situation with upsample filter. The stopband attenuation of the upsample filter for this worst case situation is sufficient to meet with the specifications.

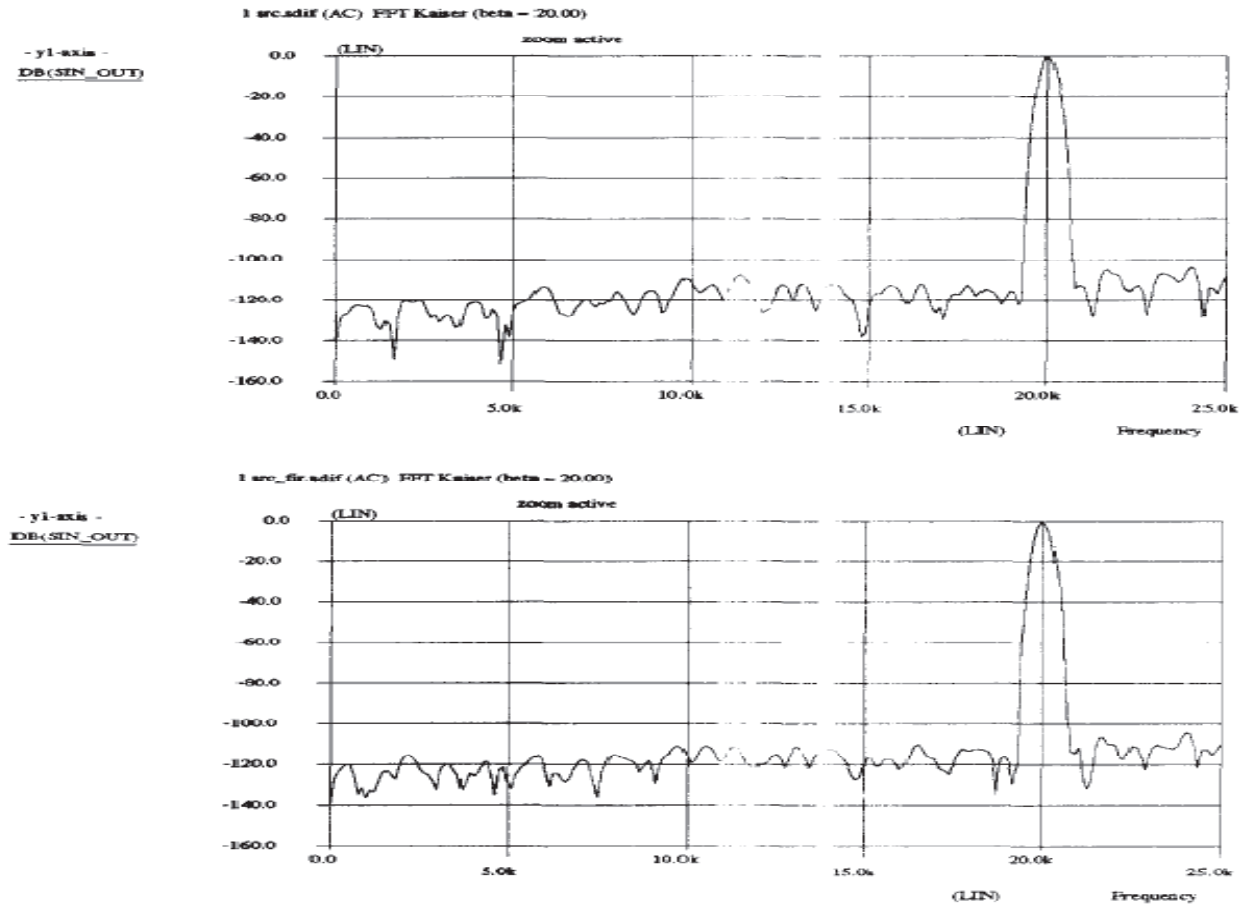

Fig. 3 Frequency domain results of the sample-rate converter without upsample filter (above) and with upsample filter (below).

\section{Conclusions}

In this thesis a technique of digital sample-rate conversion is described and analyzed from a theoretical point of view, at system level. With this technique, the output samples are obtained by the taking over, the omission or the repetition of the input samples. This new method of samplerate conversion can be used for the interconnection of two audio devices having a different sampling frequency, as well as for digital mixing of a few sources having a different sampling frequency.

The sample-rate converter based on shaping of time errors requires an upsample filter which is much simpler than those used in conventional digital sample-rate converters. The upsampling factor can be lower and the stopband attenuation can therefore be smaller. The computational complexity needed is much smaller than for conventional sample-rate conversion methods. This gives a reduction in the nedded hardware. As a conclusion it can be stated that the specifications of the upsample filter depend on the situation for which the sample-rate converter is used.

\section{REFERENCES}

[1] Adams R. W., (1986) "Design and implementation of an audio 18 bit analog-to-digital converter using oversampling techniques”, J.Audio Engineering Society,34(3), 153-166. 
[2] Agrawal B. P. and Shenoi K.(1983) "Digital methodology for $\sum \Delta \mathrm{M}$ ”, IEEE Trans. Communications, 31(3), 360-370.

[3] R. Crochiere and L. Rabiner, (1983) Mullinale Digital Signal Processing., Englewood Cliffs, NJ: Prentice Hall.

[4] Therssen, D., (1990) "A new Principle for sample-rate conversion of digital signals", Leuven, Belgium.

[5] Baggen, C.P.M.J., (1993) An Information Theoretic Approach to Timing Jitter, Doctoral Dissertation, University of California, San Diego.

[6] R. Fitzgerald and W. Anderson, (1992) "Spectral distortion in sampling rate conversion by zero-order polynomial interpolation”, IEEE Trans. Signal Processing, vol. 40, pp. 1576-1578.

[7] H.G. Göckler, G. Evangelista, and A. Groth, (2001) "Minimal Polyphase Implementation of Fractional Sample Rate Conversion”, Signal Processing, vol. 81, no.4, pp. 673-691.

[8] Groth, and H.G. Göckler, (2001) "Efficient Minimum Group Delay Block Processing Approach to Fractional Sample Rate Conversion”, ISCAS '01, Sydney, Australia, vol. II, pp. 189-192.

[9] H.G. Göckler, G. Evangelista, and A. Groth, "Minimal Polyphase Implementation of Fractional Sample Rate Conversion,” Signal Processing, vol. 81, no.4, pp. 673-691, April 2001.

[10] Baggen, C.P.M.J., An Information Theoretic Approach to Timing Jitter, Doctoral Dissertation, University of California, San Diego, 1993.

[11] Heidemann, G.H.L. Mand Taltje, H.E.P., Stochastic Signal Theory, College Reader, University of Twente, the Netherlands, 1991.

[12] Therssen, D., “ A phase locked loop for the new sample rate converter", Philips ADC Leuven, Belgium, 1992.

[13] Nuijten, P.A.C.M., Asynchronous D/A conversion, Nat. Lab Personal Note, Philips Research Laboratories, Eindhoven, 1994.

\section{Authors}

Mr. Manish Sabraj received his Bachelor's degree in Electronics and Communication Engineering from University of Jammu. He received his M.Tech degree in Electronics and Communication Engineering from IIT Guwahati in year 2003. He worked as Lecturer during period 2004-2008. Presently, he is working as Asstt. Professor in School of Electronics and Communication Engineering, Shri Mata Vaishno Devi University, India. His research interests include digital signal processing, control system and digital communication.

Dr. Vipan Kakkar received his Bachelor's and Master's degree in Electrical Engineering. He received his doctorate in VLSI from Delft University of Technology, Netherlands in 2002. During 2001/2-2008, he worked in Netherlands in VLSI design for audio and video applications. He worked in Netherlands for 8 years at different positions. Presently, he is working as Director in school of Electronics and Communications Engineering at SMVD University, India. His research interests include digital signal processing, Video Processing in VLSI.
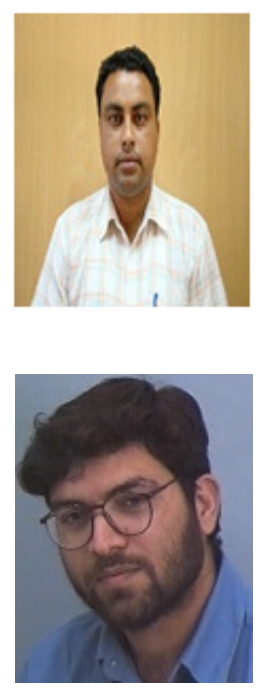